\title{
Contrast of time-averaged images of the solar granulation
}

\author{
P. N. Brandt ${ }^{1}$ and A. V. Getling ${ }^{2}$ \\ ${ }^{1}$ Kiepenheuer-Institut für Sonnenphysik, Schöneckstrasse 6, 79104 Freiburg, Germany, \\ email: pnb@kis.uni-freiburg.de \\ ${ }^{2}$ Institute of Nuclear Physics, Lomonosov Moscow State University, Moscow, 119992, Russia, \\ email: A.Getling@ru.net
}

\begin{abstract}
The time-averaged images of the solar granulation exhibit a slower decrease in contrast with the averaging time than do time-averaged images of numerically simulated granulation and time-averaged random fields with some parameters typical of granulation. This confirms the hints for long-lived structures in the granulation pattern.
\end{abstract}

As we reported before (Getling \& Brandt 2002a, b), the procedure of time averaging applied to the 11-h La Palma series of granulation images (see Simon et al. 1994 for a description) had revealed a conspicuously slow decrease in the rms contrast with the averaging time; this may be a hint for long-lived photospheric structures. In his comment on this observation, Rast (2002) claimed that the contrast variation with the averaging time does not change substantially if the real images are replaced by artificially created, random fields with some characteristic parameters typical of granulation. On this basis, Rast raised doubts on the reality of the long-lived component of the granulation dynamics.

Rast's analysis of the contrast variation was not convincing since the fairly wide spread in the contrast of individual solar images prevented accurate comparisons. In this study, to obtain unambiguous results, we subjected the images to a certain standardization procedure. Specifically, we renormalized not only the mean intensity but also the rms intensity contrast of the images to a certain specific value, which was the same for all images analysed. In addition, we removed the residual mean-intensity gradients within the frame (which were notable in the images of the La Palma series).

Upon the standardization, we computed the variation of the rms contrast with the averaging time $t$ (at a fixed mid-time of the averaging interval and a total averaging time of about $7 \mathrm{~h}$ ) for the following four series of images: (1) the real (La Palma) granulation images within a $43.5 \times 43.5 \mathrm{Mm}^{2}$ area, (2) the artificial granulation patterns obtained by Rieutord et al. (2002) in their numerical simulations of granular-scaled convection and covering a $30 \times 30 \mathrm{Mm}^{2}$ area, (3) a similar series computed by M. Steffen, B. Freytag, and H.-G. Ludwig (see Wedemeyer et al. 2004) for an $11.2 \times 11.2 \mathrm{Mm}^{2}$ area, and (4) Rast's random fields corresponding to a $38.4 \times 38.4 \mathrm{Mm}^{2}$ area.

The resulting contrast-variation curves are shown in the figure, on both linear and loglog scales. The rms contrast of an individual image is normalized to unity in all cases. It can be seen that the curve for the solar images runs well above the three other curves. It diverges most widely with the curve for Rast's random field, while the curves for the numerically simulated granulation remain in between these extremes. The differences in the slopes are most pronounced in the region of $t \lesssim 1 \mathrm{~h}$, but remain quite appreciable within the entire range of averaging times. This can be noted most easily if we compare the slopes of the curves with that of the straight line representing (in the right-hand graph) a statistical $t^{-1 / 2}$ law, which should be expected for completely random fields at 

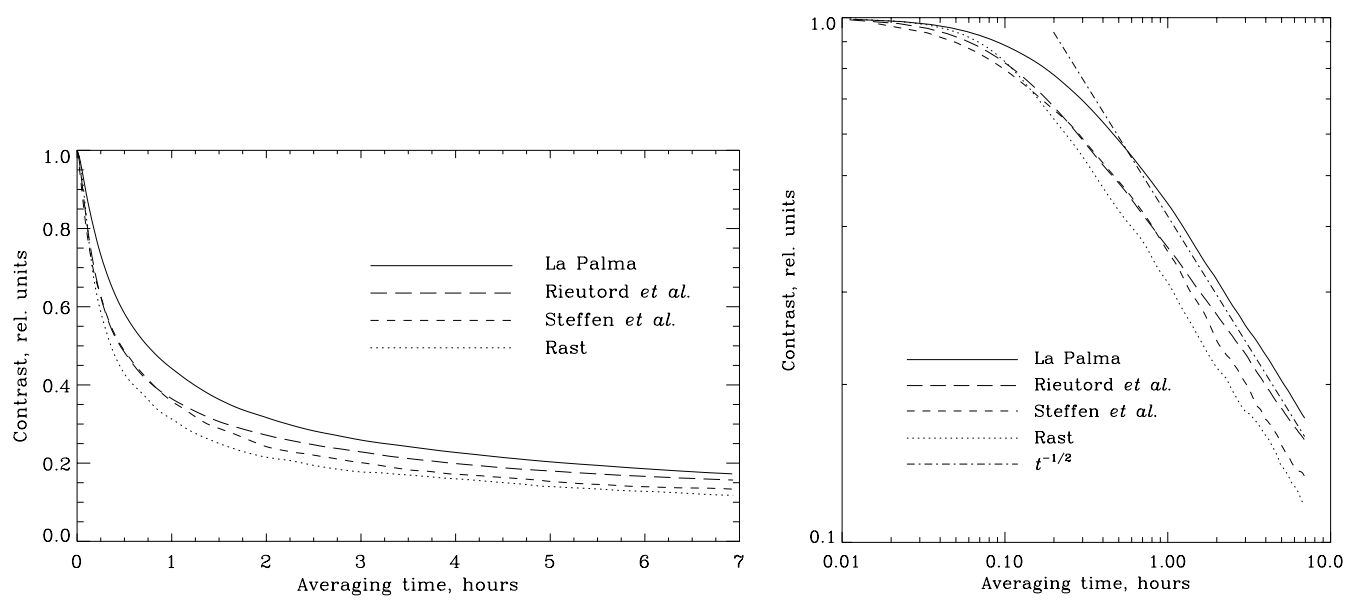

Contrast of the averaged images as a function of the averaging time for the four datasets considered, on linear (left) and log-log (right) scales. In the right graph, a $t^{-1 / 2}$ law of variation is also shown for comparison.

sufficiently large $t$. The curve for the real granulation remains sloping more gently than the $t^{-1 / 2}$ line even in the rightmost part of the graph, where $t \approx 7 \mathrm{~h}$. At the same time, the curve for Rast's field is virtually parallel to the $t^{-1 / 2}$ line for $t \gtrsim 1 \mathrm{~h}$. A rapid decline can also be noted for the images simulated by Steffen et al. As for the images obtained by Rieutord et al. (2002) in their simulations, the corresponding curve also runs well below that of the La Palma series, although it exhibits, for $t \gtrsim 1 \mathrm{~h}$, the slowest decline among the four curves considered and even approaches the La Palma curve at large $t$.

We see that the contrast of the solar images exhibits a markedly different behaviour compared to the simulated granulation and to the random fields, especially at short to moderate averaging times. Thus, the solar granulation pattern seems to evolve more slowly than the artificial fields do and possibly contains a long-lived component.

\section{Acknowledgements}

We are grateful to T. Roudier, M. Steffen, and M. P. Rast for making their data available to us. The work of A. V. G. was supported by the Deutsche Forschungsgemeinschaft and by the Russian Foundation for Basic Research (project code 04-02-16580).

\section{References}

Getling, A. V. \& Brandt, P. N. 2002a Astron. Astrophys. 382, L5-L8.

Getling, A. V. \& Brandt, P. N. 2002b In Proc. 10th European Solar Physics Meeting, 'Solar Variability: From Core to Outer Frontiers'. Prague, Czech Republic, 9-14 September 2002. ESA SP-506, pp. 617-620.

Getling, A. V. 2004 In Multi-Wavelength Investigations of Solar Activity, Proc. IAU Symp. No. 223. St Petersburg, 14-19 June 2004 (ed. A. V. Stepanov, E. E. Benevolenskaya \& A. G. Kosovichev), pp. ??-??. Cambridge University Press.

Rast, M. P. 2002 Astron. Astrophys. 392, L13-L15.

Rieutord, M., Ludwig, H.-G., Roudier, T., Nordlund, Å. \& Stein, R. 2002 Nuovo Cimento 25, 523-529.

Simon, G. W., Brandt, P. N., November, L. J., Scharmer, G. B. \& Shine, R. A. 1994 In Solar Surface Magnetism, Proc. Workshop. Soesterberg, The Netherlands, 1-5 November 1993 (ed. R. J. Rutten \& C. J. Schrijver), pp. 261-270. Kluwer.

Wedemeyer, S., Freytag, B., Steffen, M., Ludwig, H.-G., \& Holweger, H. 2004 Astron. Astrophys. 414, 1121-1137. 